\title{
APROVEITAMENTO PELA CANA-DE-AÇÚCAR DA ADUBAÇÃO NITROGENADA DE PLANTIO(1)
}

\author{
Henrique Coutinho Junqueira Franco ${ }^{(2)}$, Paulo Cesar Ocheuze Trivelin ${ }^{(3)}$, Carlos \\ Eduardo Faroni ${ }^{(4)}$, André Cesar Vitti ${ }^{(5)} \&$ Rafael Otto ${ }^{(6)}$
}

RESUMO

\begin{abstract}
$\mathrm{O}$ balanço de ${ }^{15} \mathrm{~N}$ de fontes nitrogenadas no sistema solo-planta tem sido de muita valia em estudos das transformações do $\mathrm{N}$ em diferentes agroecossistemas. No agrossistema da cana-de-açúcar, nas condições brasileiras, a resposta à adubação nitrogenada de cana-planta ainda é questão não totalmente esclarecida e a utilização de fertilizantes nitrogenados marcados $\operatorname{com}{ }^{15} \mathrm{~N}$ pode auxiliar no entendimento dessa lacuna. Com o objetivo de avaliar o aproveitamento do $\mathrm{N}$ da uréia pela cana-deaçúcar no ciclo agrícola de cana-planta, realizaram-se dois experimentos em área comercial de cana-de-açúcar, com o cultivar SP81-3250. Esses experimentos foram feitos de fevereiro de 2005 a julho de 2006. O delineamento experimental foi o de blocos ao acaso, sendo os tratamentos constituídos de três doses de N: 40,80 e $120 \mathrm{~kg}$ $h^{-1}$, na forma de uréia, e uma testemunha sem fertilização nitrogenada. No centro das parcelas com doses de $\mathrm{N}$-uréia, foram instaladas microparcelas que receberam o fertilizante marcado com ${ }^{15} \mathrm{~N}$. A recuperação de ${ }^{15} \mathrm{~N}$-uréia pela cana-planta (planta toda) foi na média dos experimentos de 30,30 e $21 \%$, respectivamente, para as doses de 40, 80 e $120 \mathrm{~kg} \mathrm{ha}^{-1}$ de $\mathrm{N}$. A menor recuperação do N-uréia nas maiores doses, especialmente na de $120 \mathrm{~kg} \mathrm{ha}^{-1}$, deveu-se às perdas de $\mathrm{N}$ do sistema solo-planta. $\mathrm{O}$ aproveitamento do $\mathrm{N}$ da uréia $\left({ }^{15} \mathrm{~N}\right)$ representou em média $11,7 \%$ do $\mathrm{N}$ total acumulado na planta toda. A distribuição do $\mathrm{N}$ proveniente do fertilizante nas diversas partes da planta não variou com a dose de $\mathrm{N}$, sendo em média de $50 \%$ nos colmos, $22 \%$ nas folhas secas, $20 \%$ nos ponteiros e $8 \%$ nas raízes.
\end{abstract}

Termos de indexação: Saccharum spp., uréia, ${ }^{15 N}$, cana-planta.

\footnotetext{
(1) Parte do projeto de doutorado do primeiro autor e parte do projeto Temático processo $\mathrm{n}^{\mathrm{o}}$ 02/10534-8 (FAPESP). Trabalho apresentado no XXXI Congresso Brasileiro de Ciência do Solo, 2007 (Gramado, RS)

(2) Pós-Doutor, Bolsista FAPESP, Laboratório de Isótopos Estáveis, Centro de Energia Nuclear na Agricultura (CENA-USP). Av. Centenário, 303, Caixa Postal 96, Piracicaba-SP, CEP 13400-970. E-mail: hjfranco@cena.usp.br

(3) Professor Associado, Bolsista CNPq, Laboratório de Isótopos Estáveis, Centro de Energia Nuclear na Agricultura (CENAUSP). E-mail: pcotrive@cena.usp.br

(4) Pesquisador do Centro de Tecnologia Canavieira (CTC), Faz. Santo Antônio s/ noำ, Bairro Santo Antônio, Caixa Postal 162, Piracicaba-SP, CEP 13400-970. E-mail: cfaroni@ctc.com.br

(5) Pesquisador Científico, Agência Paulista de Tecnologia dos Agronegócios, Pólo Regional Centro Sul. Rodovia SP 127, km 30, Vila Fátima, Piracicaba-SP, CEP 13400-970. E-mail: acvitti@apta.sp.gov.br

(6) Doutorando do Programa de Pós-Graduação em Solos e Nutrição de Plantas da Escola Superior de Agricultura "Luiz de Queiroz" (ESALQ-USP). Bolsista FAPESP. Laboratório de Isótopos Estáveis, CENA/USP. E-mail: rotto@esalq.usp.br
} 


\title{
SUMMARY: UTILIZATION BY SUGAR CANE OF NITROGEN APPLIED AT PLANTING
}

\begin{abstract}
The ${ }^{15} \mathrm{~N}$ balance of $\mathrm{N}$ sources in the soil-plant system is of great value in studies of $N$ transformation in different agroecosystems. In the sugarcane agroecosystem, the cane plant response to $\mathrm{N}$ fertilization for Brazilian conditions is not fully understood and the use of $N$ fertilizer labeled with ${ }^{15} \mathrm{~N}$ can help clarify this issue. To evaluate the urea- $N$ utilization by sugarcane at plant cane harvest, $t$ wo experiments were developed with the variety SP81-3250, in commercial sugarcane fields. The experiments were carried out from February 2005 to July 2006. The experiment was a randomized complete block design and the treatments three $N$-urea rates $\left(40,80\right.$ and $\left.120 \mathrm{~kg} \mathrm{ha}^{-1}\right)$ and a control without $N$-fertilization $\left(0 \mathrm{~kg} \mathrm{ha} \mathrm{a}^{-1} \mathrm{~N}\right)$. In the center of the plots with urea application microplots were installed and treated with ${ }^{15} \mathrm{~N}$-labeled urea. The average recovery (\%) of $15 \mathrm{~N}$-fertilizer by sugarcane (whole plant) was 30,30 and $21 \%$, respectively, at rates of 40,80 and $120 \mathrm{~kg} \mathrm{ha}^{-1}$ of $\mathrm{N}$. The lower urea-N recovery at higher rates, mainly for $120 \mathrm{~kg}$ $\mathrm{ha}^{-1}$ of $N$, was due to $N$ losses from the soil-plant system. The $N$-urea recovery was on average $11.7 \%$ of the total accumulated $N$ in the whole plant. The different $N$ rates on $N$ from fertilizer did not influence $N$ distribution in the different sugarcane plant parts, which were on average $50 \%$ in the stalks, $22 \%$ in the dry leaves, $20 \%$ in the shoots and $8 \%$ in the roots.
\end{abstract}

Index terms: Saccharum spp, urea, ${ }^{15 N}$, plant cane.

\section{INTRODUÇÃO}

O N apresenta uma dinâmica complexa, pelas múltiplas transformações caracterizadas por sete estados de oxidação e por sua mobilidade no sistema solo-planta. Os fertilizantes nitrogenados aplicados ao solo sofrem uma série de transformações químicas e microbianas, que podem resultar em perdas para os vegetais. Nesse contexto, considerando o custo dos adubos nitrogenados, é fundamental o desenvolvimento de manejos adequados da adubação nitrogenada que visem ao melhor aproveitamento do $\mathrm{N}$ pela cultura da cana-de-açúcar. Com isso, a realização do balanço de ${ }^{15} \mathrm{~N}$ de fontes nitrogenadas tem sido de muita valia em estudos das transformações do $\mathrm{N}$ no sistema solocana-de-açúcar.

Trabalhos realizados com fertilizantes nitrogenados marcados com o isótopo ${ }^{15} \mathrm{~N}$ evidenciaram ser variável 0 aproveitamento do ${ }^{15} \mathrm{~N}$-fertilizante pela cultura da cana-de-açúcar. Nas Ilhas Maurícios, Wong You Cheong et al. (1980) obtiveram recuperação na parte aérea da cana-de-açúcar de 21 a $48 \%$, com as fontes sulfato de amônio e nitrato de amônio, respectivamente, com influência do tipo de solo, das condições climáticas e da fonte nitrogenada. Ng Kee Kwong \& Deville (1994) conseguiram aumentar a recuperação do ${ }^{15} \mathrm{~N}$-uréia $\left(120 \mathrm{~kg} \mathrm{ha}^{-1}\right)$ de 19 para cerca de $35 \%$, aplicando o adubo na água e irrigando por gotejamento, sem, contudo, conseguir elevar a produtividade. Em Taiwan, Weng et al. (1991) obtiveram as recuperações de 27,23 e $19 \%$, respectivamente, para sulfato de amônio, nitrato de potássio e uréia; a aplicação dos adubos no solo a $10 \mathrm{~cm}$ de profundidade, comparada à superficial, mostrou a maior recuperação. Na Austrália, Chapman et al. (1994) obtiveram recuperação de ${ }^{15} \mathrm{~N}$-uréia para a planta toda, em três sistemas de manejo com resíduos culturais no solo, de 18 e $33 \%$, para o adubo aplicado em superfície e em profundidade, respectivamente. No Brasil, Bittencourt et al. (1986), Sampaio et al. (1984), Gava et al. (2001) e Trivelin et al. (1995, 1996, 2002a,b) obtiveram recuperação de fertilizantes nitrogenados (sulfato de amônio, uréia e aquamônia) de 0,2 a 54 \%.

Essas variações na recuperação do ${ }^{15} \mathrm{~N}$ fertilizante pela cultura de cana-de-açúcar podem estar associadas, ainda, ao efeito residual do fertilizante no solo, considerando sua elevada imobilização (Courtaillac et al., 1998) e as perdas do $\mathrm{N}$ no sistema solo-planta, tais como as perdas por desnitrificação (Trivelin et al., 2002a), lixiviação (Oliveira et al., 1999), volatilização de amônia (Trivelin et al., 2002a), bem como as perdas gasosas de $\mathrm{N}$ pela parte aérea das plantas (Holtan-Hartwing \& Bockman, 1994).

No entanto, nas condições brasileiras, os resultados de recuperação do ${ }^{15} \mathrm{~N}$-fertilizante pela cana-de-açúcar, obtidos no ciclo de cana-planta em condições de campo, são incipientes. Portanto, é importante avaliar o aproveitamento do ${ }^{15} \mathrm{~N}$-fertilizante pela cana-planta em experimentos de campo, principalmente pelo fato de ser a adubação nitrogenada em cana-planta uma das questões ainda não esclarecidas no manejo dos canaviais e, com isso, a utilização de fertilizantes nitrogenados marcados com ${ }^{15} \mathrm{~N}$ auxiliaria no entendimento dessa lacuna. E, ainda, com a futura 
obrigatoriedade na colheita sem despalha a fogo, o balanço de ${ }^{15} \mathrm{~N}$, realizado nos compartimentos da planta: folhas secas, colmo, ponteiro e raízes, fornecerá informações mais exatas sobre a reciclagem do ${ }^{15} \mathrm{~N}$ fertilizante no sistema solo-planta desde a instalação do canavial (cana-planta).

Nesse contexto, o objetivo deste trabalho foi avaliar 0 aproveitamento do ${ }^{15} \mathrm{~N}$-uréia e a distribuição do $\mathrm{N}$ em plantas de cana-de-açúcar (cana-planta) colhida sem queima.

\section{MATERIAL E MÉTODOS}

O experimento foi desenvolvido em duas áreas comerciais de reforma do canavial. A primeira área é pertencente à Usina São Luiz (Pirassununga-SP), cujo solo foi classificado como Latossolo Vermelho-Amarelo distrófico. A análise de terra para fins de fertilidade foi realizada nas profundidades de 0-25 cm e 25-50 $\mathrm{cm}$, antes da instalação do experimento, tendo a análise química apresentado: $\mathrm{pH} 5,5$ e 4,7 em $\mathrm{CaCl}_{2}$; 9 e $6 \mathrm{mg} \mathrm{dm}^{-3}$ de P (resina); $\mathrm{K}, \mathrm{Ca}, \mathrm{Mg}, \mathrm{H}+\mathrm{Al}, \mathrm{CTC}$, Al: 2,4 e $1,0 \mathrm{mmol}_{\mathrm{c}} \mathrm{dm}^{-3}, 29$ e $10 \mathrm{mmol}_{\mathrm{c}} \mathrm{dm}^{-3}, 8$ e 3 $\mathrm{mmol}_{\mathrm{c}} \mathrm{dm}^{-3}, 20$ e $31 \mathrm{mmol}_{\mathrm{c}} \mathrm{dm}^{-3}, 20$ e $31 \mathrm{mmol}_{\mathrm{c}} \mathrm{dm}^{-3}$ respectivamente; m igual a 2,5 e $18 \%$ e V de 66 e $31 \%$. As principais operações no preparo do solo antes do plantio foram: gradeação pesada para matar a soqueira antiga; subsolagem para quebrar a compactação do solo; gradeação pesada para incorporação de corretivos ( $2 \mathrm{Mg}^{\text {ha }}{ }^{-1}$ de calcário dolomítico e $2 \mathrm{Mg} \mathrm{ha}^{-1}$ de gesso agrícola) e gradeação média para preparo final do solo antes da sulcação. A instalação do experimento ocorreu entre 21 e 24 de fevereiro de 2005.

A segunda área foi instalada na Usina Santa Adélia (Jaboticabal-SP), em um Latossolo Vermelho-Escuro, apresentando as seguintes características químicas, respectivamente, nas profundidades de $0-25 \mathrm{~cm}$ e 25 50 cm: pH 5,1 e 4,2 em $\mathrm{CaCl}_{2} ; 15$ e $5 \mathrm{mg} \mathrm{dm}^{-3}$ de $\mathrm{P}$ (resina); K, Ca, Mg, H+Al, CTC, Al: 3,9 e 1,8 mmol $_{\mathrm{c}}$ $\mathrm{dm}^{-3}, 11$ e $14 \mathrm{mmol}_{\mathrm{c}} \mathrm{dm}^{-3}, 5$ e $1 \mathrm{mmol}_{\mathrm{c}} \mathrm{dm}^{-3}, 28$ e 34 $\mathrm{mmol}_{\mathrm{c}} \mathrm{dm}^{-3}$, 1 e $8 \mathrm{mmol}_{\mathrm{c}} \mathrm{dm}^{-3}$, respectivamente; $\mathrm{m}$ igual a 4,8 e $54 \%$ e V de 42 e $17 \%$. As principais operações efetuadas na reforma do canavial antes do plantio foram: dessecação da soqueira velha com aplicação de $4 \mathrm{~L} \mathrm{ha}^{-1}$ de Roundup; aração profunda para incorporação ao solo dos resíduos vegetais e de 2 $\mathrm{Mg}$ ha $^{-1}$ de calcário dolomítico e gradeação para o preparo final do solo antes da sulcação e plantio. A instalação do experimento deu-se entre 4 e 8 de abril de 2005.

O delineamento experimental empregado foi o de blocos ao acaso com quatro repetições. Os tratamentos foram três doses de $\mathrm{N}\left(40,80\right.$ e $120 \mathrm{~kg} \mathrm{ha}^{-1}$ na forma de uréia) aplicadas manualmente no fundo do sulco de plantio, mais uma testemunha sem aplicação de N. O fertilizante nitrogenado foi incorporado ao solo. As parcelas experimentais foram compostas por 48 linhas (sulcos) de $15 \mathrm{~m}$ espaçadas entre si por 1,5 m. No plantio, foram aplicados no fundo de sulco, em todas as parcelas, o cloreto de potássio e o superfosfato simples nas doses de $120 \mathrm{~kg} \mathrm{ha}^{-1}$, respectivamente, de $\mathrm{K}_{2} \mathrm{O}$ e $\mathrm{P}_{2} \mathrm{O}_{5}$. No interior de cada parcela, onde foi aplicado o fertilizante nitrogenado, instalou-se uma microparcela, com dimensão de $2 \mathrm{~m}$ de comprimento e $1,5 \mathrm{~m}$ de largura, totalizando $3 \mathrm{~m}^{2}$, que recebeu a uréia marcada com ${ }^{15} \mathrm{~N}\left(5,04 \%\right.$ em átomos de $\left.{ }^{15} \mathrm{~N}\right)$. Os adubos foram cobertos manualmente com terra antes do plantio da cana-de-açúcar.

No plantio da cana-de-açúcar, cruzou-se o pé com a ponta das mudas (colmos), proporcionando uma distribuição de 17 a 20 gemas por metro linear de sulco. As mudas de cana depositadas no fundo do sulco foram cortadas em toletes com 2 a 3 gemas e feito o recobrimento com máquina. $\mathrm{O}$ cultivar de cana-deaçúcar plantado foi o SP81-3250.

A colheita final foi realizada entre 7 e 10 de junho e 11 a 13 de julho de 2006, respectivamente, nos experimentos das Usinas São Luiz e Santa Adélia. A colheita da parte aérea das plantas das microparcelas com ${ }^{15} \mathrm{~N}$-uréia e também na testemunha, sem adubação nitrogenada, foi realizada manualmente em $1 \mathrm{~m}$ de linha, no centro e em posições contíguas nas linhas adjacentes à microparcela, separando-se amostras de folhas secas, ponteiros e colmos. Nessas amostras, foi determinada a massa de material vegetal natural. Todo o material foi triturado em picadora mecânica de forragem. Depois da moagem e homogeneização de cada amostra úmida, retirou-se uma subamostra, que foi seca em estufa $\left(65^{\circ} \mathrm{C}\right)$, sendo determinada a umidade desse material. $\mathrm{O}$ material seco foi moído em moinho Willey e usado nas determinações de $\mathrm{N}$-total e de abundância de ${ }^{15} \mathrm{~N}(\%$ em átomos de ${ }^{15} \mathrm{~N}$ ) no espectrômetro de massa ANCA/ SL, modelo 20/20 da Europa Scientific, Krewe, U.K.

Após a colheita da parte aérea das plantas de canade-açúcar das microparcelas, foi realizada a amostragem de raízes, obtidas mediante o uso da sonda Bravifer ( $55 \mathrm{~mm}$ de diâmetro interno) no centro das microparcelas. Essa amostragem foi efetuada na profundidade de $0-60 \mathrm{~cm}$, segundo Faroni \& Trivelin (2006). As amostras de solo e raízes obtidas por sondagem nas microparcelas com ${ }^{15} \mathrm{~N}$-uréia foram embaladas em sacos plástico, identificadas adequadamente e transportadas ao CENA, onde foi feita a separação do solo das raízes por peneiramento e a seco (malha da peneira - $2 \mathrm{~mm}$ ). As raízes e rizomas separados do solo foram lavados em água corrente, secos em estufa ventilada a $65^{\circ} \mathrm{C}$ e obtidas as massas de material seco, em seguida realizou-se a moagem desse material em moinho tipo Willey. Essas amostras foram submetidas às determinações de $\mathrm{N}$ total e de abundância de ${ }^{15} \mathrm{~N}$ por espectrometria de massas.

A recuperação $(\mathrm{RN})$ do ${ }^{15} \mathrm{~N}$-fertilizante na planta foi calculada por meio das equações:

$$
\mathrm{NPPF}=[(\mathrm{A}-\mathrm{C}) /(\mathrm{B}-\mathrm{C})] \cdot \mathrm{NT}
$$




$$
\mathrm{RN}(\%)=(\mathrm{NPPF} / \mathrm{NAF}) 100
$$

significando: $\mathrm{RN}$ - recuperação percentual do ${ }^{15} \mathrm{~N}$ fertilizante na planta; NPPF - N na planta proveniente do ${ }^{15} \mathrm{~N}$-fertilizante; A - abundância de ${ }^{15} \mathrm{~N}$ (\% de átomos) da planta; B - abundância de ${ }^{15} \mathrm{~N}$ (5,04 \% de átomos) do N-fertilizante; C - abundância natural de ${ }^{15} \mathrm{~N}(0,366 \%$ de átomos); NT - conteúdo de N na planta $\left(\mathrm{kg} \mathrm{ha}^{-1}\right)$; NAF - dose de $\mathrm{N}$ da fonte aplicada $\left(\mathrm{kg} \mathrm{ha}^{-1}\right)$.

Os resultados foram submetidos à análise de variância, utilizando o teste $\mathrm{F}$ a $90 \%$ de confiança. Para comparar o efeito de doses de N, usou-se a análise de regressão polinomial.

O quadro 1 apresenta os valores de precipitação pluvial mensais ocorridos durante o período experimental.

\section{RESULTADOS E DISCUSSÃO}

A recuperação do ${ }^{15} \mathrm{~N}$-uréia $\left(\mathrm{kg} \mathrm{ha}{ }^{-1}\right)$, nas partes da planta no ensaio da Usina Santa Adélia (USA), foi significativamente maior nos tratamentos em que se empregaram as doses mais elevadas de N (Quadro 2), exceção feita ao ponteiro, em que não houve efeito significativo. Esse fato deveu-se a possíveis perdas de $\mathrm{N}$ pela parte aérea das plantas durante o processo de maturação fisiológica, haja vista que, além da recuperação do ${ }^{15} \mathrm{~N}$-uréia no ponteiro ter sido semelhante entre os tratamentos, o acúmulo de $\mathrm{N}$, nessa parte da planta, foi menor nas doses de 80 e $120 \mathrm{~kg} \mathrm{ha}^{-1} \mathrm{de}$ N (Quadro 3).

Quadro 1. Precipitações pluviais mensais nas áreas experimentais nas Usinas Santa Adélia (USA) e São Luiz (USL)

\begin{tabular}{|c|c|c|c|c|c|c|c|c|c|c|c|c|c|c|c|c|c|c|c|}
\hline \multirow{3}{*}{ Local } & \multicolumn{19}{|c|}{ Precipitação pluvial mensal } \\
\hline & \multicolumn{11}{|c|}{2005} & \multicolumn{7}{|c|}{2006} & \multirow[b]{2}{*}{ Total } \\
\hline & Fev & Mar & Abr & Mai & Jun & Jul & Ag & Set & Out & Nov & Dez & Jan & Fev & Mar & Abr & Mai & Jun & Jul & \\
\hline USA & -- & -- & 87 & 82 & 33 & 40 & 0,0 & 85 & 105 & 27 & 166 & 229 & 450 & 244 & 7,0 & 0,0 & 13 & 0,0 & 1568 \\
\hline USL & 20 & 192 & 33 & 57 & 11 & 18 & 1,0 & 56 & 79 & 56 & 233 & 177 & 320 & 202 & 53 & 3,0 & 0,0 & -.- & 1511 \\
\hline
\end{tabular}

Quadro 2. Recuperação do N-uréia $\left(\mathrm{kg} \mathrm{ha}^{-1}\right)$ pela cana-de-açúcar na colheita nos experimentos das Usinas Santa Adélia e São Luiz

\begin{tabular}{lllll}
\hline Doses de N & Colmo & Folha seca & Ponteiro & Parte aérea
\end{tabular}

$\mathrm{kg} \mathrm{ha}^{-1}$

\begin{tabular}{|c|c|c|c|c|c|c|}
\hline \multicolumn{7}{|c|}{ Usina Santa Adélia } \\
\hline 40 & 6,1 & 3,2 & 2,2 & 11,5 & 0,8 & 12,3 \\
\hline 80 & 9,7 & 5,9 & 3,1 & 18,7 & 1,3 & 20,0 \\
\hline 120 & 13,5 & 6,7 & 3,2 & 23,4 & 1,5 & 24,9 \\
\hline Teste F & $5,37^{*}$ & $2,37^{*}$ & $1,02^{\mathrm{NS}}$ & $5,22^{*}$ & $3,58 * *$ & $5,74^{*}$ \\
\hline $\mathrm{R}^{2}-$ R.L. & $0,99^{*}$ & $0,92^{* *}$ & NS & $0,99^{*}$ & $0,95^{*}$ & $0,98^{*}$ \\
\hline $\mathrm{R}^{2}-\mathrm{R} \cdot \mathrm{Q}$. & NS & NS & NS & NS & NS & NS \\
\hline C.V. $(\%)$ & 32,7 & 40,5 & 40,5 & 29,5 & 35,0 & 28,0 \\
\hline \multicolumn{7}{|c|}{ Usina São Luiz } \\
\hline 40 & 6,1 & 2,0 & 2,7 & 10,8 & 1,4 & 12,2 \\
\hline 80 & 14,5 & 3,9 & 7,4 & 25,8 & 2,2 & 28,0 \\
\hline 120 & 12,0 & 5,4 & 5,5 & 22,9 & 2,5 & 25,4 \\
\hline Teste F & $13,3^{*}$ & $11,5^{*}$ & $7,66^{* *}$ & $25,51^{*}$ & $1,03^{\mathrm{NS}}$ & $28,14^{*}$ \\
\hline $\mathrm{R}^{2}$ - R.L. & $0,46^{*}$ & $0,99^{*}$ & NS & $0,58^{*}$ & NS & $0,61^{*}$ \\
\hline $\mathrm{R}^{2}-\mathrm{R} \cdot \mathrm{Q}$. & $0,99^{*}$ & NS & $0,99 *$ & $0,99 *$ & NS & $0,99 *$ \\
\hline C.V. (\%) & 21,9 & 27,2 & 32,7 & 15,9 & 56,6 & 14,7 \\
\hline
\end{tabular}

NS: não significativo; * $\mathrm{e}^{* *}$ significativos, respectivamente, a 5 e $10 \%$. 
Quadro 3. Acúmulo de nitrogênio nas partes das plantas de cana-de-açúcar na colheita da cultura nos experimentos das Usinas Santa Adélia e São Luiz

\begin{tabular}{|c|c|c|c|c|c|c|}
\hline Doses de N & Colmo & Folha seca & Ponteiro & Parte aérea & Raiz & Planta toda \\
\hline & \multicolumn{6}{|c|}{ Usina Santa Adélia } \\
\hline $\mathrm{kg} \mathrm{ha}^{-1}$ & \multicolumn{6}{|c|}{$\mathrm{N}$ total, $\mathrm{kg} \mathrm{ha}^{-1}$} \\
\hline 0 & 72,9 & 30,4 & 40,8 & 144,1 & 8,4 & 152,5 \\
\hline 40 & 102,1 & 36,6 & 40,7 & 179,4 & 12,6 & 192,1 \\
\hline 80 & 89,0 & 36,1 & 33,6 & 160,1 & 9,8 & 170,0 \\
\hline 120 & 90,7 & 35,6 & 28,2 & 154,5 & 12,0 & 166,5 \\
\hline Teste F & $1,93^{\mathrm{NS}}$ & $0,80^{\mathrm{NS}}$ & $3,19^{* *}$ & $1,54^{\mathrm{NS}}$ & $2,04^{\mathrm{NS}}$ & $1,64^{\mathrm{NS}}$ \\
\hline $\mathrm{R}^{2}-$ R.L. & NS & NS & $0,90^{*}$ & NS & NS & NS \\
\hline $\mathrm{R}^{2}-\mathrm{R} \cdot \mathrm{Q}$. & NS & NS & NS & NS & NS & NS \\
\hline \multirow[t]{2}{*}{ C.V. (\%) } & 19,4 & 18,7 & 19,1 & 15,0 & 25,7 & 15,0 \\
\hline & \multicolumn{6}{|c|}{ Usina São Luiz } \\
\hline 0 & 64,6 & 17,7 & 37,5 & 119,8 & 7,2 & 127,1 \\
\hline 40 & 81,8 & 22,2 & 44,5 & 147,8 & 9,4 & 157,3 \\
\hline 80 & 97,1 & 28,0 & 52,2 & 177,4 & 10,2 & 187,5 \\
\hline 120 & 77,8 & 24,9 & 35,7 & 138,4 & 12,7 & 151,1 \\
\hline Teste F & $2,99 * *$ & $3,18^{* *}$ & $3,33^{* *}$ & $5,01^{*}$ & $1,83^{\mathrm{NS}}$ & $5,6^{*}$ \\
\hline $\mathrm{R}^{2}-\mathrm{R} . \mathrm{L}$ & NS & $0,65^{*}$ & NS & NS & $0,96^{*}$ & $0,28^{*}$ \\
\hline $\mathrm{R}^{2}-\mathrm{R} . \mathrm{Q}$ & $0,90^{*}$ & NS & $0,82^{*}$ & $0,86^{*}$ & NS & $0,88^{*}$ \\
\hline C.V. (\%) & 19,3 & 21,1 & 19,4 & 14,7 & 33,8 & 13,5 \\
\hline
\end{tabular}

NS: não-significativo; * $\mathrm{e}$ ** significativos, respectivamente, a 5 e $10 \%$.

Na senescência foliar, durante o período de maturação fisiológica, o aumento da fotorrespiração e da hidrólise de proteínas foi acompanhado pela redução nas atividades das enzimas glutamina sintetase (GS) e glutamato sintase (GOGAT), principais responsáveis pela assimilação da amônia $\left(\mathrm{NH}_{3}\right)$ no metabolismo do $\mathrm{N}$ nas plantas superiores. A redução na atividade dessas enzimas resultou no aumento da concentração de $\mathrm{NH}_{4}{ }^{+}$nas células das plantas (Mattsson et al., 1998). Como o $\mathrm{NH}_{4}^{+}$em altas concentrações é tóxico para os vegetais (Holtan-Hartwing \& Bockman, 1994; Mattsson et al., 1998), esta redução pode resultar em perdas naturais de $\mathrm{NH}_{3}$ junto à corrente transpiratória.

Os resultados de recuperação $\left(\mathrm{kg} \mathrm{ha}^{-1}\right)$ obtidos no ensaio da Usina São Luiz (USL) foram semelhantes aos observados na USA, ou seja, aumento na recuperação do ${ }^{15} \mathrm{~N}$-uréia com o emprego de maiores doses de $\mathrm{N}$ (Quadro 2). No entanto, no ponteiro e no colmo, ocorreram reduções na recuperação do tratamento com aplicação de $120 \mathrm{~kg} \mathrm{ha}^{-1}$ de N. Os dados obtidos nessas partes da planta (ponteiro e colmo) contribuíram para menor recuperação do ${ }^{15} \mathrm{~N}$-uréia na parte aérea e planta toda da cana-de-açúcar (Quadro 2).

Os decréscimos observados na recuperação do ${ }^{15} \mathrm{~N}$ uréia no experimento da USL são corroborados pelos resultados de acúmulo de N (Quadro 3), tendo em vista que na maior dose de $\mathrm{N}\left(120 \mathrm{~kg} \mathrm{ha}^{-1}\right)$ obteve-se o menor acúmulo de $\mathrm{N}$ em relação ao da dose de $80 \mathrm{~kg} \mathrm{ha}^{-1}$ de $\mathrm{N}$, em todas as partes da planta, excluindo-se as raízes, onde não houve diferença entre os tratamentos.

Os reduzidos valores de recuperação do ${ }^{15} \mathrm{~N}$-uréia, nos dois experimentos, especialmente na maior dose, podem ter como causa as perdas de ${ }^{15} \mathrm{~N}$-fertilizante durante o ciclo da cultura. Essas perdas podem ter ocorrido por lixiviação de $\mathrm{NO}_{3}{ }^{-}$, desnitrificação, volatilização de amônia do fertilizante e por perdas naturais de $\mathrm{N}$ pela folhagem da cultura durante $\mathrm{o}$ processo de maturação.

As perdas de $\mathrm{N}$ por lixiviação podem ser desconsideradas, pois, na maioria dos ensaios com o emprego de ${ }^{15} \mathrm{~N}$ em cana-de-açúcar, constatou-se que essas perdas são pequenas. Bologna-Campbell (2007) não verificou perdas mensuráveis por lixiviação de $\mathrm{N}$ derivado do fertilizante $\left({ }^{15} \mathrm{~N}\right)$. O N total lixiviado médio correspondeu a $13,1 \mathrm{mg}$ vaso $^{-1}$, equivalendo a $0,44 \mathrm{~kg} \mathrm{ha}^{-1}$ de $\mathrm{N}$, sendo originado, provavelmente, do $\mathrm{N}$ nativo do solo. No trabalho de Ng Kee Kwong \& Deville (1984), desenvolvido em lisímetros cultivados com cana-de-açúcar, não ocorreram perdas de $\mathrm{N}$ por lixiviação, mesmo em condições de elevada precipitação pluvial média anual, 1.300 e $3.200 \mathrm{~mm}$, em duas regiões das Ilhas Maurício. $\mathrm{O}$ fato foi atribuído à 
imobilização microbiana e ao movimento mais lento de $\mathrm{NO}_{3}{ }^{-}$em relação ao da água percolada. Oliveira et al. (1999) também não constataram perdas de $\mathrm{N}$ por lixiviação, utilizando a técnica do ${ }^{15} \mathrm{~N}$. Aliado a isso, na área da USA, Ghiberto et al. (2007) monitoraram o fluxo de água no solo no ciclo da cana-planta mediante a utilização de tensiômetros e extratores de solução do solo, constataram que a lixiviação de $\mathrm{N}$ foi de $15 \mathrm{~kg} \mathrm{ha}^{-1}$, porém, as perdas de ${ }^{15} \mathrm{~N}$ proveniente do fertilizante foram desprezíveis, sendo de $21 \mathrm{~g} \mathrm{ha}^{-1}$.

Outra via de saída de $\mathrm{N}$ está ligada a perdas por desnitrificação do $\mathrm{NO}_{3}$ - derivado do fertilizante, o que pode ter ocorrido, principalmente, no primeiro mês após a adubação (março) no experimento da USL, em decorrência de elevada precipitação pluvial (Quadro 1), aliada à incorporação ao solo de resíduos culturais do ciclo anterior e ao consumo de $\mathrm{O}_{2}$ pelos microrganismos (quimiorganotróficos anaeróbios facultativos), o que possivelmente proporcionou condições de anaerobiose. No experimento da USA, acredita-se que este fenômeno não tenha ocorrido, pois as precipitações pluviais (Quadro 1) foram menos intensas nos primeiros meses após a adubação nitrogenada (abril e maio).

A volatilização de amônia após a hidrólise da uréia pode ser desconsiderada, tendo em vista o modo de aplicação do ${ }^{15} \mathrm{~N}$-uréia (no fundo do sulco de plantio seguido de incorporação ao solo). Segundo Trivelin et al. (2002a), quando a uréia é incorporada ao solo numa profundidade entre 15 e $25 \mathrm{~cm}$, as perdas de $\mathrm{NH}_{3}$ por volatilização são desprezíveis.

Portanto, a principal perda de $\mathrm{N}$ do sistema soloplanta pode ser atribuída à volatilização de amônia pela parte aérea das plantas, conforme discutido anteriormente. As perdas de N pela parte aérea de canade-açúcar foram estimadas, indiretamente, por Ng Kee Kwong \& Deville (1984) e Trivelin (2002a), respectivamente, nas Ilhas Maurício e no Brasil, como sendo da ordem de $100 \mathrm{~kg} \mathrm{ha}^{-1} \mathrm{ano}^{-1}$, de mesma grandeza das doses de $\mathrm{N}$ aplicadas nas fertilizações de canaviais.

Nos dois locais, a distribuição do $\mathrm{N}$ proveniente do fertilizante nas diversas partes da planta não variou com a dose de N. Na USL, essa distribuição foi a seguinte: $50 \%$ nos colmos, $17 \%$ nas folhas secas, $23 \%$ nos ponteiros e $10 \%$ nas raízes, aproximadamente (Quadro 2). Na USA (Quadro 2), a distribuição aproximouse da anterior, sendo: $27 \%$ do ${ }^{15} \mathrm{~N}$-fertili-zante encontrado nas folhas secas, $16 \%$ nos ponteiros, $6 \%$ nas raízes e o restante nos colmos (51\%). Os resultados obtidos nas folhas secas indicam que a absorção do ${ }^{15} \mathrm{~N}$ uréia no ensaio da USA foi mais rápida do que na USL, independentemente de o fertilizante ter sido aplicado 45 dias após a aplicação feita na USL, sendo isso devido, possivelmente, a melhores condições de crescimento do sistema radicular da cana-planta no experimento da USA (melhor ambiente de produção). Trivelin et al. (2002b) e Bologna-Campbell (2007) constataram, no momento da colheita da cana-planta cultivada em lisímetros, que cerca de $40 \%$ do ${ }^{15} \mathrm{~N}$-fertilizante absorvido pela cana-de-açúcar estava nas folhas secas, $22 \%$ na parte subterrânea, $19 \%$ nos colmos e $15 \%$ nos ponteiros.
Segundo os autores, o maior acúmulo de $\mathrm{N}$ derivado do fertilizante nas folhas secas da planta de cana-de-açúcar foi devido à maior absorção do ${ }^{15} \mathrm{~N}$-fertilizante nos estádios iniciais de desenvolvimento da cultura.

Por outro lado, os resultados de distribuição do ${ }^{15} \mathrm{~N}$-uréia (Quadro 2) e do $\mathrm{N}$ acumulado na planta (Quadro 3) mostram o benefício da colheita sem despalha a fogo, tendo em vista que cerca de $20 \%$ do $\mathrm{N}$ total e do ${ }^{15} \mathrm{~N}$-uréia na planta toda estavam contidos nas folhas secas, quantidades estas que seriam perdidas para atmosfera pela queima do canavial antes da colheita.

O N absorvido da uréia representou em média 11,1 e $12,3 \%$ do acumulado na planta toda, respectivamente, na USA e USL. Sampaio et al. (1984) avaliaram que a contribuição do $\mathrm{N}$ da uréia para as doses de 20 e $60 \mathrm{~kg} \mathrm{ha}^{-1}$ de $\mathrm{N}$ foi menos que $10 \%$ do total acumulado na planta toda de cana-de-açúcar. Trivelin et al. (2002b) obtiveram um valor de 11,5\% para as doses de $\mathrm{N}$ de 30,60 e $90 \mathrm{~kg} \mathrm{ha}^{-1}$ aplicados na forma de uréia; Gava et al. (2001) verificaram que o N na planta proveniente do fertilizante representou de 10 a $16 \%$ do $\mathrm{N}$ total acumulado na parte aérea da soqueira de cana-de-açúcar, enquanto Trivelin et al. (1995) obtiveram um valor menor que $15 \%$ em cana-soca de fim de safra. No entanto, Bologna-Campbell (2007) constatou uma contribuição mais elevada, variando de 9,5 a $27,2 \%$, com resposta linear para as doses de $\mathrm{N}$ (40, 80 e $\left.120 \mathrm{~kg} \mathrm{ha}^{-1}\right)$, em todas as partes da planta.

Com base nos resultados da literatura e nos deste trabalho, pode-se afirmar que independentemente das condições experimentais, o $\mathrm{N}$ na planta proveniente do ${ }^{15} \mathrm{~N}$-fertilizante quantificado na colheita representa pequena fração do $\mathrm{N}$ total acumulado pela cultura de cana-de-açúcar. Como efeito, as principais fontes de $\mathrm{N}$ para a cultura de cana-de-açúcar no ciclo de canaplanta devem ter sido a mineralização da matéria orgânica nativa do solo e de resíduos culturais ( $\mathrm{Ng}$ Kee Kwong et al., 1987; Sampaio et al., 1995), e a fixação biológica do $\mathrm{N}_{2}$ atmosférico por microrganismos (Urquiaga et al., 1992). Entretanto, não deve ser desconsiderada a hipótese de maior aproveitamento do ${ }^{15} \mathrm{~N}$-uréia durante o ciclo de crescimento da planta, considerando a ocorrência de perdas de $\mathrm{N}$ pela parte aérea, conforme mencionado anteriormente.

Em relação à recuperação percentual, os dados obtidos na USA não apresentaram diferenças significativas entre si dentro de cada compartimento de planta, exceção observada no ponteiro onde ocorreu redução na recuperação com o aumento da dose de $\mathrm{N}$ (Quadro 4). Já na USL, as reduções foram verificadas em todos os compartimentos, exceto nas raízes e ponteiros (Quadro 4). Sampaio et al. (1984) constataram a redução na percentagem de recuperação do ${ }^{15} \mathrm{~N}$-fertilizante com o aumento das doses de N, mesmo com o aumento nos valores absolutos de recuperação $\left(\mathrm{kg} \mathrm{ha}^{-1}\right)$. Muitas vezes, a redução na recuperação percentual do ${ }^{15} \mathrm{~N}$-fertilizante com o aumento das doses é justificada pelas perdas do $\mathrm{N}$ aplicado no sistema solo-planta, conforme discutido anteriormente. 
Quadro 4. Recuperação do N-uréia na colheita da cultura de cana-de-açúcar nos experimentos das Usinas Santa Adélia e São Luiz

\begin{tabular}{|c|c|c|c|c|c|c|}
\hline Doses de N & Colmo & Folha seca & Ponteiro & Parte aérea & Raiz & Planta toda \\
\hline \multicolumn{7}{|l|}{$\mathrm{kg} \mathrm{ha} \mathrm{a}^{-1}$} \\
\hline & \multicolumn{6}{|c|}{ Usina Santa Adélia } \\
\hline 40 & 15,2 & 8,1 & 5,4 & 28,8 & 1,9 & 30,6 \\
\hline 80 & 12,1 & 7,3 & 3,9 & 23,4 & 1,6 & 25,0 \\
\hline 120 & 11,3 & 5,6 & 2,7 & 19,5 & 1,3 & 20,8 \\
\hline Teste F & $0,89^{\mathrm{NS}}$ & $1,33^{\mathrm{NS}}$ & $3,85^{* *}$ & $1,87^{\mathrm{NS}}$ & $1,19^{\mathrm{NS}}$ & $2,05^{\mathrm{NS}}$ \\
\hline $\mathrm{R}^{2}-$ R.L. & NS & NS & $0,99^{*}$ & NS & NS & $0,99^{* *}$ \\
\hline $\mathrm{R}^{2}-\mathrm{R} . \mathrm{Q}$ & NS & NS & NS & NS & NS & NS \\
\hline \multirow[t]{2}{*}{ C.V. (\%) } & 34,4 & 31,6 & 35,0 & 28,6 & 35,5 & 27,1 \\
\hline & \multicolumn{6}{|c|}{ Usina São Luiz } \\
\hline 40 & 15,2 & 4,9 & 6,8 & 26,9 & 3,4 & 30,3 \\
\hline 80 & 18,2 & 4,8 & 9,3 & 32,2 & 2,7 & 35,0 \\
\hline 120 & 10,0 & 4,5 & 4,6 & 19,1 & 2,1 & 21,1 \\
\hline Teste F & $6,90^{*}$ & $0,16^{\mathrm{NS}}$ & $6,34^{*}$ & $13,47^{*}$ & $0,95^{\mathrm{NS}}$ & $13,43^{*}$ \\
\hline $\mathrm{R}^{2}-$ R.L. & $0,39^{*}$ & NS & NS & $0,35^{*}$ & NS & $0,43^{*}$ \\
\hline $\mathrm{R}^{2}-\mathrm{R} . \mathrm{Q}$. & $0,99^{*}$ & NS & $0,99^{*}$ & $0,99^{*}$ & NS & $0,99^{*}$ \\
\hline C.V. (\%) & 21,9 & 22,9 & 27,0 & 13,9 & 50,7 & 13,3 \\
\hline
\end{tabular}

${ }^{\mathrm{NS}}$ : não-significativo; ${ }^{*} \mathrm{e}{ }^{* *}$ significativos, respectivamente, a 5 e $10 \%$.

A recuperação (\%) de ${ }^{15} \mathrm{~N}$-uréia pela canaplanta (planta toda) na média dos experimentos foi de 30,30 e $21 \%$, respectivamente, para as doses de 40, 80 e $120 \mathrm{~kg} \mathrm{ha}^{-1}$ de N (Quadro 4). Esses valores de recuperação estão de acordo com os resultados da literatura para condições de campo, cerca de 10 a 50 \% (Wong You Cheong et al., 1980; Sampaio et al., 1984; Weng et al., 1991; Chapman et al., 1994; Trivelin et al., 1995, 1996; Gava et al., 2001).

\section{CONCLUSÕES}

1. A recuperação do ${ }^{15} \mathrm{~N}$-uréia pela cana-planta (planta toda) na média dos experimentos foi de 30,30 e $21 \%$, respectivamente, para as doses de 40,80 e $120 \mathrm{~kg} \mathrm{ha}^{-1}$ de N.

2. O N absorvido da uréia representou em média $11,7 \%$ do $\mathrm{N}$ total acumulado na planta toda.

3. A distribuição do $\mathrm{N}$ proveniente do fertilizante nas diversas partes da planta não variou com a dose de $\mathrm{N}$ empregada, sendo, em média, de: $50 \%$ nos colmos, $22 \%$ nas folhas secas, $20 \%$ nos ponteiros e $8 \%$ nas raízes.

\section{LITERATURA CITADA}

BITTENCOURT, V.C.; FAGANELLO, B.F. \& SALATA, J.C. Eficiência da adubação nitrogenada em cana-de-açúcar (planta). STAB - Açúcar, Álcool Subpr., 5:26-33, 1986.

BOLOGNA-CAMPBELL, I. Balanço de nitrogênio e enxofre no sistema solo-cana-de-açúcar no ciclo de cana-planta. Piracicaba, Escola Superior de Agricultura Luiz de Queiroz, 2007. 112p. (Tese de Doutorado)

CHAPMAN, L.S.; HAYSOM, M.B.C. \& SAFFIGNA, P.G. The recovery of ${ }^{15} \mathrm{~N}$ from labelled urea fertilizer in crop components of sugarcane and in soil profiles. Austr. J. Agric. Res., 45:1577-1585, 1994.

COURTAILLAC, N.; BARAN, R.; OLIVER, R.; CASABIANCA, H. \& GANRY, F. Efficiency of nitrogen fertilizer in sugarcane-vertical system in Guadeloupe according to growth and ratoon age of the cane. Nutr.Cycling Agroecosyst., 52:9-17, 1998.

FARONI, C.E. \& TRIVELIN, P.C.O. Quantificação de raízes metabolicamente ativas de cana-de-açúcar. Pesq. Agropec. Bras., 41:1007-1013, 2006.

GAVA, G.J.C.; TRIVELIN, P.C.O.; OLIVEIRA, M.W. \& PENATTI, C.P. Crescimento e acúmulo de nitrogênio em cana-de-açúcar cultivada em solo coberto com palhada. Pesq. Agropec. Bras., 36:1347-1354, 2001. 
GHIBERTO, P.J.; LIBARDI, P.L.; BRITO, A.S. \& TRIVELIN, P.C.O. Lixiviação de nitrogênio em um Latossolo cultivado com cana-de-açúcar. In: CONGRESSO BRASILEIRO DE CIÊNCIA DO SOLO, 31., Gramado, 2007. Anais. Gramado, 2007. CD-ROM.

HOLTAN-HARTWIG, L. \& BOCKMAN, O.C. Ammonia exchange between crops and air. Norwegian J. Agric. Sci.14S:1-41, 1994. (Supplement)

MATTSSON, M.; HUSTED, S. \& SCHJOERRING, J.K. Influence of nutrition and metabolism on ammonia volatilization in plants. Nutr. Cycling Agroecosyst., 51:35-40, 1998.

NG KEE KWONG, K.F. \& DEVILLE, J. Nitrogen leaching from soils cropped with sugarcane under the humid tropical climate of Mauritius, Indian Ocean. J. Environ. Quality, 13:471-474, 1984.

NG KEE KWONG, K.F.; DEVILLE, J.; CAVALOT, P.C. \& RIVIERE, V. Value of cane trash in nitrogen nutrition of sugarcane. Plant Soil, 102:79-83, 1987.

NG KEE KWONG, K.F. \& DEVILLE, J. Application of ${ }^{15} \mathrm{~N}$ labelled urea to sugar cane through a drip-irrigation system in Mauritius. Fert. Res., 39:223-228, 1994.

OLIVEIRA, M.W.; TRIVELIN, P.C.O.; GAVA, G.J.C.; VITTI, A.C. Lixiviação de nitrogênio em solo cultivado com canade-açúcar: experimento em lisímetro. STAB - Açúcar, Álcool Subpr., 18:28-31, 1999.

SAMPAIO, E.V.S.B.; SALCEDO, I.H. \& BETTAMY, J Dinâmica de nutrientes em cana-de-açúcar. I. Eficiência de utilização de uréia- ${ }^{15} \mathrm{~N}$ em aplicação única ou parcelada. Pesq. Agropec. Bras., 19:943-949, 1984.

SAMPAIO, E.V.S.B.; SALCEDO, I.H.; SILVA, V.M. \& ALVES, G.D. Capacidade de suprimento de nitrogênio e resposta à fertilização de vinte solos de Pernambuco. R. Bras. Ci. Solo, 19:269-279, 1995.
TRIVELIN, P.C.O.; VICTORIA, R.L. \& RODRIQUES, J.C. Aproveitamento por soqueira de cana-de-açúcar de final de safra do nitrogênio da aquamônia- ${ }^{15} \mathrm{~N}$ e aplicado ao solo em complemento à vinhaça. Pesq. Agropec. Bras., 30:1375-1385, 1995.

TRIVELIN, P.C.O.; VICTORIA, R.L. \& RODRIQUES, J.C. Utilização por soqueira de cana-de-açúcar de início de safra do nitrogênio da aquamônia- ${ }^{15} \mathrm{~N}$ e uréia- ${ }^{15} \mathrm{~N}$ aplicado ao solo em complemento à vinhaça. Pesq. Agropec. Bras., 31:89-99, 1996.

TRIVELIN, P.C.O.; OLIVEIRA, M.W.; VITTI, A.C.; GAVA, G.J.C. \& BENDASSOLLI, J.A. Perdas do nitrogênio da uréia no sistema solo-planta em dois ciclos de cana-deaçúcar. Pesq. Agropec. Bras., 37:193-201, 2002a.

TRIVELIN, P.C.O.; VITTI, A.C.; OLIVEIRA, M.W.; GAVA, G.J.C. \& SARRIÉS, G.A. Utilização de nitrogênio e produtividade da cana-de-açúcar (cana-planta) em solo arenoso com incorporação de resíduos da cultura. R. Bras. Ci. Solo, 37:193-201, 2002b.

URQUIAGA, S.; CRUZ, K.H.S. \& BODDEY, R.M. Contribution of nitrogen fixation to sugar cane: nitrogen-15 and nitrogen-balance estimates. Soil Sci. Soc. Am. J., 56:105$114,1992$.

WENG, T.; CHAN, Y. \& LI, S. Effects of various forms of nitrogen fertilizers and application methods on sugarcane yield and nitrogen uptake. Taiwan Sugar, 38:22-24, 1991.

WONG YOU CHEONG, Y.; NG KEE KWONG, K.F. \& CAVALOT, P.C. Comparative study of ammonium and nitrate fertilizers in two soils of Mauritius cropped with sugar-cane. In: RESEARCH COORDINATION MEETING IN SOIL NITROGEN AS FERTILIZER OR POLLUTANT, 1978. Proceedings. Vienna, IAEA, 1980. p.351-367. 